\title{
HL-A Linked Genetic Control of Immune Response in Man
}

\author{
Michael J. HaVerkorn, ${ }^{1}$ B. Hofman, ${ }^{2}$ N. MASUREL ${ }^{3} \&$ J. J. VAN ROOD ${ }^{4}$
}

After the original observation by Gorer \& Schütze (1938) that some breeds of mice were more susceptible to particular infections than other breeds, research in this field has given many arguments for genetic control of immune response to a variety of antigens in animals (McDevitt \& Benacerraf 1969). In several instances this control has been shown to be linked to loci controlling histocompatibility antigens in mouse, guinea pig or rat (Benacerraf 1974, McDevitt et al. 1974a, McDevitt \& Bodmer 1974). This article presents the preliminary results of a study of immune response in human twins.

If a pair of sibs is HL-A identical and if the genetic control of immune response is associated with loci located on the same chromosome carrying the loci responsible for HL-A antigens, one can expect a greater similarity in immune response than in a pair of sibs which is not HL-A identical. Thus, greater homogeneity in immune response in HL-A identical sibs is considered an argument in favour of linkage between some immune response locus and the HL-A loci.

For the first part of our study we chose antibody titres to antigens in vaccines or common infectants as parameters for antibody response in man. There are reports on associations between immune response and particular HL-A haplotypes, but these are not informative in choosing an antigen for linkage studies (Jersild et al. 1973, Levine et al. 1972). Antibody responses to poliomyelitis virus and diphtheria toxoid were chosen because they are included in routinely administrated vaccinations of children in the Nether-

1 Laboratory of Epidemiology, Department of Microbial Diseases, University Hospital, Leiden;

2 Laboratory of Poliomyelitisvaccin, Central Public Health Institute, Bilthoven;

3 Department of Virology, Erasmusuniversity, Rotterdam;

4 Department of Immunohematology, University Hospital, Leiden, the Netherlands. 
lands, and these vaccinations are well documented in municipal files. Antibody responses to rubella, measles and influenza were included because these are common infectious diseases among children in the Netherlands, usually affecting a whole family if one member is infected, and lead to antibody titers detectable for long periods of times thereafter.

To limit the interference of differences in other, non-genetic factors in immune response, only twin pairs of equal sex, living in the same household, and attending the same elementary school in Leiden or the Hague were studied. Zygosity was determined according to some obvious anthropologic measurements, such as eye colour, and on the basis of seven redcell antigen systems. Twin pairs were considered dizygotic if they differed in at least two of these parameters.

Twins of each pair were examined on the same day. This examination was postponed if there was any doubt about the state of health of either twin on that day. Antibodies against measles were tested for in two haemagglutination inhibition tests systems, using different antigens (Brouwer et al. 1974).

\section{RESULTS}

Out of 154 twin pairs examined, 11 were excluded on the basis of inconclusive evidence for dizygosity. As the parameter for the degree of similarity of antibody response within a twin pair, the ratio between the highest and the lowest antibody titre was used. Harmonic means of these ratios are tabulated in Table I. These harmonic means are shown to illustrate differences; they are not involved in the statistical analysis of these differences. Included are 71 monozygotic and 72 dizygotic twin pairs. In this latter group of twin pairs, 18 share no HL-A haplotypes, 31 share only one HL-A haplotype, and 23 share both HL-A haplotypes.

Distributions of these ratios were tested for statistical evidence of difference by the Wilcoxon two-sample test for analysis of the data on poliomyelitis, diphtheria and influenza. The chi-square test was used for testing independence in a two-way classification of data for measles and rubella. If there was no convincing evidence that vaccination of both twin and cotwin had been done on the same day, the twin pair was included in a separated group of twin pairs having different vaccination dates. The levels of statistical significance tabulated are those for two-sided tests.

\section{DISCUSSION}

Comparison of antibody titres between mono and dizygotic twins, regard- 
less of HL-A haplotypes, indicates the relative contribution of genetic factors to these antibody titres, and hence to immune response. In these data there is statistically significant evidence only for antibodies against measles, poliomyelitis I virus, and diphtheria toxoid. The latter is of special importance because it appears in both of the mutually-independent samples, that of twin pairs with similar vaccination dates and that of twin pairs with different vaccination dates.

If linkage between HL-A and 'immune response' does exist, it can only lower the ratios of HL-A identical twin pairs as compared to the ratios of HL-A non-identical twin pairs. This difference is not according to expectation for all antibody titre ratios tested. It is so for the haemagglutinating antibodies against measles in both test systems, however. The level of statistical significance is under 5 per cent in a one-sided test for one of them, and not far above 5 per cent for the other test system.

In comparison with the data from animal studies, it is not surprising to find these differences between the results with the various antigens. In animal studies great variation in results with different antigens are found also (Benacerraf 1974, McDevitt \& Benacerraf 1969). The indication in favour of linkage between HL-A loci and some locus or loci responsible for factors affecting the antibody response to measles is strong enough to warrant further pursuit. This is done by continuing this study and extending it to the study of antibody responses to recent vaccinations using sera taken at fixed intervals after vaccination.

\section{SUMMARY}

In a study of 143 pairs of school-age twins, a significant contribution of genetic factors in the immune response to measles as well as in the immune response to diphtheria toxoid are shown. This is in contrast to the immune responses to poliomyelitis vaccine, rubella and influenza virus. In a study of 72 dizygotic twin pairs among them, evidence was shown of a possible linkage between HL-A loci and loci involved in the immune response against measles. Similar evidence was not found for antibody responses against other antigens in the same twins.

\section{REFERENCES}

Benacerraf, B. (1974) The genetic mechanisms that control the immune response and antigen recognition. Ann. Immunol. (Inst. Pasteur) 125 C, 143.

Brouwer, R., de Groot, I. G. M. \& Verheij, F. B. M. (1974) Comparison of maternal and cord serum titres for measles and for rubella antibodies. Arch. ges. Virusforsch. 44, 237. 
Gorer, P. A. \& Schütze, H. (1938) Genetical studies on immunity in mice. II. Correlation between antibody formation and resistance. J. Hygiene 38, 647.

Jersild, C., Svejgaard, A., Fog, T. \& Amnitzbøll, T. (1973) HL-A antigens and diseases. I. Multiple sclerosis. Tissue Antigens 3, 243.

Levine, B. B., Stember, R. H. \& Fotino, M. (1972) Ragweed hay fever: Genetic control and linkage to HL-A haplotype. Science 178, 1201.

McDevitt, H. O. \& Benacerraf, B. (1969) Genetic control of specific immune response. Advanc. Immunol. 11, 31.

McDevitt, H. O., Oldstone, M. B. A. \& Pincus, Th. (1974) Histocompatibility-linked genetic control of specific immune responses to viral infections. Transplant. Rev. 19, 209.

McDevitt, H. O. \& Bodmer, W.F. (1974) HL-A, immune-response genes and disease. Lancet i, 1269. 
This document is a scanned copy of a printed document. No warranty is given about the accuracy of the copy. Users should refer to the original published version of the material. 